\title{
CAMBIOS EN LA GESTIÓN INSTITUCIONAL EN UNIVERSIDADES, A PARTIR DE LA IMPLEMENTACIÓN DEL SISTEMA NACIONAL DE ASEGURAMIENTO DE LA CALIDAD: LA EXPERIENCIA CHILENA ${ }^{1}$
}

\author{
María José Lemaitre, Mario Maturana, Elisa Zenteno, \\ Andrea Alvarado
}

\section{RESUMEN}

La paulatina instalación del Sistema Nacional de Aseguramiento de Calidad en la Educación Superior y, principalmente, los procesos de acreditación institucional, han producido impactos en la gestión de las instituciones de nuestro país. Estos cambios se observan especialmente en la gestión del aseguramiento de la calidad, en términos de creación o adaptación de estructuras orgánicas, de mecanismos, uso y análisis de insumos de información, buenas prácticas y, en general, un mayor grado de familiaridad de los actores involucrados. Esta investigación tiene por objeto identificar dichos impactos, precisando los cambios y las buenas prácticas, con la finalidad de contribuir con dicho conocimiento al mejoramiento de la gestión de las instituciones de educación terciaria.

Palabras clave: calidad en educación superior, aseguramiento de la calidad, gestión de la calidad en la educación superior

\section{CHANGES IN INSTITUTIONAL MANAGEMENT IN UNIVERSITIES SINCE THE IMPLEMENTATION OF A QUALITY ASSURANCE NATIONAL SYSTEM: THE CHILEAN EXPERIENCE}

\section{ABSTRACT}

The gradual implementation of a National System of Quality Assurance in Higher Education and, overall, the institutional accreditation processes, have produced some impacts in institutional management in our country. These changes are especially evident in quality assurance management in terms of creation and adaptation of organic structures, mechanisms, use and analysis of information inputs, good practices and, in general, a better participation of relevant actors. This research is aimed to identify such impacts, detailing changes and good practices, in order to try to improve the management of tertiary education institutions.

Keywords: higher education quality, quality assurance, management of higher education quality

1 Este estudio contó con apoyo financiero del Consejo Nacional de Educación (CNED).

2 Centro Universitario de Desarrollo, CINDA. Santiago, Chile. Contacto: mjlemaitre@cinda.cl 


\section{CAMBIOS EN LA GESTIÓN INSTITUCIONAL EN UNIVERSIDADES, A PARTIR DE LA IMPLEMENTACIÓN DEL SISTEMA NACIONAL DE ASEGURAMIENTO DE LA CALIDAD: LA EXPERIENCIA CHILENA}

\section{Introducción}

La implementación del Sistema Nacional de Aseguramiento de Calidad en la Educación Superior comienza en Chile con la instalación de la Comisión Nacional de Acreditación de Pregrado (CNAP), en 1999, a la cual correspondió desarrollar procesos experimentales de acreditación de carreras e instituciones y proponer un sistema nacional de aseguramiento de calidad. Dicha propuesta sirvió de base a la dictación de la Ley No 20.129, que dio forma a ese sistema nacional, y atribuyó a la CNA, instalada en el año 2007, la acreditación institucional. Ambas comisiones han dirigido los procesos de acreditación institucional y contribuido sustancialmente a la implementación del sistema nacional de aseguramiento de calidad en educación superior, así como a su incorporación en la práctica cotidiana de las instituciones.

En nuestro país no existen aún suficientes estudios sistemáticos que den cuenta de los efectos que ha tenido la acreditación en instituciones de educación superior (IES), por lo que no hay evidencia disponible que identifique mecanismos o prácticas que favorezcan una eficiente gestión de la calidad al interior de las instituciones. Esta falta de conocimiento sistematizado revela la necesidad de contar con más y mejor información respecto de los efectos que se generan con la implementación de estos mecanismos. A partir de esto, será posible identificar las experiencias que han resultado más determinantes, diferenciando a su vez los elementos y características de las que depende dicho éxito, en pos de transferir esta experiencia y contribuir a la calidad de otras instituciones de educación terciaria, mediante reflexiones y recomendaciones. 
Este estudio se propuso hacer una contribución al mejoramiento en la gestión en las IES del país, mediante la generación de conocimiento que resulte de utilidad para el aseguramiento de la calidad. Su objetivo principal fue identificar y caracterizar factores asociados a la gestión institucional que favorecen dicha mejora, para luego precisar cómo su desarrollo, en el contexto del sistema nacional de aseguramiento de calidad y específicamente los procesos de acreditación institucional, se ha expresado en acreditaciones por más tiempo.

El objetivo formulado descansa en cuatro supuestos, necesarios para dar sentido a la investigación. El primero, señala que la gestión es un ámbito que ha sido influenciado por la incorporación de mecanismos de aseguramiento de la calidad en las universidades, como ha ocurrido a lo largo de la última década. El segundo, consiste en aceptar que esta influencia ha sido positiva, ya que ha generado mejoras, tanto en la eficacia -entendida como logro de los objetivos planteados- como en la eficiencia - entendida como optimización en la utilización de recursos de la gestión de estas instituciones-. El tercer supuesto asociado es aquel que señala que los procesos de acreditación institucional constituyen los mecanismos externos de aseguramiento de calidad que han tenido mayor repercusión en la gestión universitaria. El cuarto supuesto, de carácter metodológico, se expresa en que el análisis de los cambios producidos, entre un proceso de acreditación institucional y el siguiente, constituye una forma apropiada para identificar situaciones de avance o permanencia de la capacidad para asegurar una gestión de calidad institucional.

El propósito del presente artículo es dar cuenta de los resultados de este estudio, como de la manera que estos se lograron. Para ello, se comienza señalando el estado del arte, contexto del problema y marco teórico, se sigue con la descripción del sistema nacional de aseguramiento de calidad en educación superior; su génesis, desarrollo y apreciaciones que derivan de las primeras evaluaciones del sistema. En seguida, se describe la estrategia metodológica empleada. A continuación, se hace referencia a resultados derivados de la primera y segunda etapa de la investigación, para concluir con reflexiones y recomendaciones finales. 


\section{Estado del arte y contexto del problema}

El impacto de los procesos de aseguramiento de la calidad en los sistemas de educación superior, y en particular en las instituciones que los componen, es un tema de gran interés en la actualidad. La experiencia internacional indica que existe un consenso respecto de que la aplicación de estos procesos incide de forma positiva en las instituciones, sin embargo, es difícil dilucidar sus resultados concretos o, más aún, establecer relaciones causales en cuanto a que la aplicación de dichos mecanismos genere determinados cambios en las instituciones, lo que hace prácticamente imposible medir sus consecuencias en forma directa. No obstante, es posible avanzar en esa dirección mediante aproximaciones sucesivas, en un proceso de aprendizaje compartido que permita una definición de impacto más amplia y atingente a la realidad de las IES.

Sobre la base del análisis realizado en el Taller de La Haya de 2006 (Harvey, 2006), se presenta una síntesis de los efectos que los procesos externos de aseguramiento de calidad tienen en las instituciones y programas de educación terciaria. El autor señala dos niveles en los cuales es posible identificar impactos.

El primero es el nivel de los aprendizajes. Uno de los efectos más visibles en este nivel es la valoración de los procesos de formación, observándose ajustes curriculares, establecimiento de evaluaciones diagnósticas, preocupación por los indicadores de eficacia del proceso formativo, incorporación de instancias de innovación, evaluación de aprendizajes, entre otros.

En un segundo nivel, se observan cambios en la gestión de las instituciones. Los que se hacen más evidentes y fáciles de identificar, incluyen ajustes en la declaración de la misión y propósitos institucionales, vinculación de los propósitos definidos por la institución y las políticas internas, incorporación de evidencia en procesos de toma de decisiones, vinculación entre los procesos de planificación y autoevaluación institucional, y mejoras en los sistemas de información.

En otras investigaciones realizadas, como la de Harvey y Newton (2004), se señala que el estudio de impacto del aseguramiento 
de calidad se ha orientado principalmente a determinar los efectos que la evaluación externa tiene en académicos, en procedimientos internos y estructuras de gestión. Stensaker (2008), por su parte, sintetiza los resultados de distintos estudios internacionales, señalando los ámbitos en los que parecen observarse impactos del aseguramiento de la calidad. En primer lugar, menciona cambios en las estructuras de poder al interior de las IES, señalando que los procesos de calidad generan un mayor peso en el liderazgo institucional, centralización de decisiones, mayor integración de los estudiantes a procesos de gestión interna, participación de actores del medio externo, profesionalización, mayores niveles de formalización de procedimientos internos, definición de responsabilidades, explicitación de conocimientos respecto de la calidad, así como también mayor y mejor cooperación entre académicos y entre estos y directivos. En segundo lugar, destacan los cambios en las relaciones públicas, en cuanto el impacto del mercado ha hecho que los resultados del aseguramiento de calidad se utilicen como elemento de marketing; y en tercer lugar, señala los efectos en la permeabilidad y transparencia, ya que la información se hace más transparente y accesible, lo que permite decisiones más informadas.

Finalmente, Stensaker (2008) sostiene que, a pesar de la existencia de muchos estudios sobre los impactos, aún existen serias limitaciones para identificar evidencias sustantivas con relación a la calidad de la enseñanza y aprendizaje, y calidad de la gestión en las IES. Gran parte de los estudios describen los cambios ocurridos tras la aplicación de procesos de evaluación externa organizacional (Brennan y Shah, 2000), o señalan el cumplimiento de las observaciones y recomendaciones resultantes de la evaluación (Frederiks, Westerheijden, y Weusthof, 1994).

Desde hace algunas décadas el concepto de calidad ha venido adquiriendo una centralidad explícita en el campo de la educación superior, convirtiéndose en un tema de importancia creciente, a pesar de que se reconoce que es un concepto difícil de definir, debido a su naturaleza multidimensional. Por lo mismo, son múltiples las acepciones, enfoques y acentos que ha tenido en el desarrollo de la historia. 
Muchas de las definiciones referidas a calidad se han dado en función de su evaluación, identificando estándares, criterios y/o requisitos, los cuales pueden variar según el contexto, las orientaciones y necesidades sociales preponderantes en un momento determinado.

Tal vez la definición más frecuentemente utilizada es la de ajuste a los propósitos (fitness for purpose). Sin embargo, como lo señala Lester (2005), “....una de las limitaciones del enfoque fitness for purpose es que opera por medio de la definición de objetivos y propósitos establecidos por las propias instituciones o programas académicos, sin considerar la idoneidad de los procesos en lo que se refiere a los objetivos o los requerimientos del medio externo".

Por ello, parece indispensable asociar el concepto de fitness for purpose o "ajuste a los propósitos declarados", al de fitness of purpose, es decir, "ajuste de los propósitos declarados" a las exigencias pertinentes del medio externo (tales como grupo de referencia, gobierno, agencia de aseguramiento de la calidad; disciplina; profesión; medio laboral; necesidades de los estudiantes). Una vez que estas exigencias han sido debidamente tomadas en consideración y traducidas en términos de principios y prioridades que se desprenden de la declaración de misión institucional, es posible contar con un conjunto de propósitos y fines institucionales (o de programa, según sea el caso) cuyo logro realmente es una demostración de calidad.

En este contexto y para efectos del presente estudio, se puede definir calidad en la educación superior como el grado de ajuste entre las acciones que una institución, programa académico o carrera pone en marcha para cumplir las orientaciones emanadas de su misión y propósitos institucionales, y los resultados que se obtienen de estas acciones. Ahora bien, considerando que es necesario establecer previamente la idoneidad de dichas orientaciones, la definición de propósitos institucionales debe contemplar dos dimensiones: por una parte, la consistencia externa, es decir, el ajuste a las exigencias del medio externo, expresadas en demandas provenientes del mercado laboral, la comunidad académica, o el entorno socioeconómico de cada institución; y por otra parte, la consistencia interna, que implica la traducción de dichas exigencias en función de las propias 
prioridades y principios de la institución. Así, la calidad se puede entender como la coherencia entre fines institucionales, objetivos estratégicos y actividades que se realizan para cumplir con esos objetivos, considerando además los medios que se emplean para ello.

Las buenas prácticas que se busca identificar en este estudio refieren a mecanismos y acciones instalados en IES, que facilitan el cumplimiento de su misión y propósitos de forma eficiente, es decir, mantienen o refuerzan la calidad de dichas instituciones. Con relación a este concepto, el Centro Interuniversitario de Desarrollo (CINDA) señala que las buenas prácticas se caracterizan por su grado de formalización; por la evaluación de su eficacia, es decir, tener un impacto tangible y positivo en la calidad; su sostenibilidad, porque pueden mantenerse en el tiempo; y la réplica de las mismas, ya que sirven como modelos para desarrollar políticas u otras iniciativas en otros contextos. Así, se entiende que el análisis de la gestión de la calidad en una IES permite identificar buenas prácticas con relación a ella.

\section{El sistema de aseguramiento de calidad en Chile}

\section{Génesis y desarrollo del sistema}

La preocupación por la calidad en el ámbito universitario es un tema instalado hace ya algunos años y nace como respuesta a un conjunto de trasformaciones y demandas sociales, políticas y económicas observadas en Latinoamérica en las últimas décadas, lo que ha originado la necesidad de dar cuenta de la gestión institucional y académica y el uso de recursos, obligando a las instituciones a replantearse su figura institucional y sus roles sociales. Esta realidad ha llevado a la mayoría de los países a establecer mecanismos para velar por la calidad de la educación terciaria.

En el caso chileno, la implementación de mecanismos de aseguramiento de la calidad se remonta a comienzos de la década del 90, con los procedimientos para el licenciamiento obligatorio de nuevas IES privadas (Lemaitre, 2005). A partir de esta experiencia, y luego de un amplio debate nacional sobre la creación de un sistema de acreditación, el Ministerio de Educación formó dos comisiones asesoras de evaluación 
de la calidad de programas, una para pregrado (CNAP) y otra para posgrado (CONAP). Las funciones encomendadas a estas comisiones se orientaron al diseño y aplicación de procedimientos pilotos de acreditación y a la elaboración de una propuesta de institucionalización definitiva sobre la base de la experiencia acumulada.

Al cabo de ocho años de funcionamiento, estas comisiones forjaron una interesante experiencia respecto de la implementación de mecanismos de aseguramiento de la calidad en el país, tanto de programas como de instituciones. Asimismo, fomentaron el desarrollo de una cultura de la calidad al interior de las IES, las que fueron asumiendo como una tarea propia el aseguramiento de la calidad de su gestión.

La experiencia recogida en esta aplicación piloto se concretó en la elaboración de un proyecto de ley, que fue aprobado a fines del 2006, estableciendo las bases para la implementación de un Sistema Nacional de Aseguramiento de la Calidad. Dicha ley creó la Comisión Nacional de Acreditación (CNA), organismo actualmente responsable de evaluar y verificar la calidad de los programas e IES autónomas en el país.

Desde la creación de la CNA, se ha continuado trabajando en el desarrollo de procesos de acreditación tanto institucional como de programas de pre y posgrado. Hasta diciembre del 2010, el sistema de aseguramiento de la calidad se ha pronunciado sobre la acreditación de 81 IES, de las cuales 52 son universidades y representan el 86\% de la cobertura de la matrícula autónoma de educación superior en el país (CNA, 2010a).

\section{Los procesos de acreditación institucional}

La acreditación institucional tiene por especial foco de evaluación la verificación de la existencia formal y aplicación sistemática de políticas y mecanismos para velar por el cumplimiento de los propósitos institucionales y sus resultados. Esto quiere decir: el establecimiento de un conjunto de mecanismos y políticas que regulen la calidad internamente a partir de una adecuada gestión, en los cuales se coordinen procesos permanentes de evaluación, planificación y 
monitoreo de resultados obtenidos y ajuste permanente de las actividades que realiza.

Esta perspectiva considera que las instituciones deben contar con:

- Propósitos y fines institucionales apropiados y claros, que orienten adecuadamente su desarrollo. Asimismo, las políticas y mecanismos de aseguramiento de la calidad deben velar por el cumplimiento de los propósitos declarados.

- Políticas y mecanismos de aseguramiento de la calidad que se apliquen sistemáticamente en diversos niveles institucionales, de modo eficiente y eficaz.

- Evidencia de resultados concordantes con los propósitos institucionales declarados y cautelados mediante políticas y mecanismos de autorregulación.

- Demostrar que tienen la capacidad para efectuar ajustes y cambios que mejoren su calidad y avanzar consistentemente hacia el logro de sus propósitos declarados.

Desde la perspectiva de la CNA, el mayor avance durante sus cuatro años de funcionamiento, radica principalmente en el aumento de la cobertura de procesos de acreditación, lo que se traduce en el aumento de instituciones que han obtenido su acreditación; hasta enero de 2011 existían solo dos universidades no acreditadas, una en proceso de renovación, y 53 acreditadas institucionalmente.

Tabla n. ${ }^{\circ}$ 1. Estado de las universidades chilenas respecto de la acreditación institucional, enero 2011

\begin{tabular}{|l|c|}
\hline Acreditadas & 53 \\
\hline No acreditadas & 2 \\
\hline En proceso de renovación & 1 \\
\hline Total & 56 \\
\hline
\end{tabular}

Tabla n. ${ }^{\circ}$ 2. Universidades acreditadas, según sus años de acreditación institucional, enero 2011

\begin{tabular}{|l|c|}
\hline Por 1 a 3 años & 23 \\
\hline Por 4 a 5 años & 21 \\
\hline Por 6 a 7 años & 9 \\
\hline Total & 53 \\
\hline
\end{tabular}




\section{Evaluación de la implementación de procesos de acreditación institucional}

Durante el año 2010, la agencia IPSOS realizó para la CNA un estudio para conocer la percepción del funcionamiento y los efectos del actual sistema de acreditación, para esto se encuestaron varias autoridades y directivos de IES, así como actores relevantes del sistema. Dentro de los resultados relacionados con el impacto que ha tenido la implementación de mecanismos de aseguramiento al interior de las instituciones, el estudio destaca los siguientes (IPSOS, 2010):

- La acreditación institucional es percibida como un proceso formativo y fiscalizador necesario para el adecuado funcionamiento del sistema. Asimismo, se indica que la acreditación instaura una mayor preocupación por el aseguramiento de la calidad. No obstante lo anterior, se indica que los procesos implementados han generado una sobrecarga de trabajo para la comunidad institucional en general, específicamente para quienes ocupan cargos de dirección.

- Se estima que la autoevaluación y/o evaluación interna ha permitido sistematizar los aprendizajes de las organizaciones y en la mayor parte de las instituciones se han generado planes permanentes de autoevaluación y progreso institucional, contribuyendo de esta forma en los procesos de planificación y sistematización de la información interna.

Existen otras iniciativas que apuntan también a identificar los efectos de procesos de acreditación en los sistemas de educación superior. CINDA, en colaboración con la Unión Europea, concluyó recientemente un estudio enfocado a conocer el impacto de la aplicación de mecanismos de aseguramiento de la calidad en las IES en siete países latinoamericanos y europeos; entre sus principales resultados se encuentran los siguientes puntos (CINDA, 2010):

- Según la percepción de los actores, se ha instalado una cultura de la acreditación y/o calidad que genera aprendizajes colectivos en el sistema de educación superior, incorporando instrumentos de gestión académica basados en evidencias, lo que facilita los procesos de toma de decisiones internas. Uno de los efectos más visibles es la generación de instancias institucionales para apoyar los procesos de aseguramiento de la calidad, convirtiéndose en 
elementos centrales de los procesos de gestión institucional. Se han producido ajustes y cambios en la estructura institucional, creando nuevas estructuras o reajustando otras, lo que se podría traducir en una profesionalización de procesos de gestión al interior de las IES.

- Se puede observar que la acreditación pareciera favorecer la instalación de herramientas estratégicas de gestión y planificación, lo que se traduce en una mayor eficiencia financiera, y un incremento de las confianzas entre las reparticiones institucionales.

- Se percibe cierta heterogeneidad en las respuestas de algunas instituciones a los procesos de acreditación, generando respuestas reactivas, sin que esto genere impactos en sus dinámicas internas. Existe un déficit en el desarrollo de procesos internos de aseguramiento de la calidad de manera continua y sostenida, tratándose más bien de una preocupación cíclica.

- Se ha identificado como un aspecto facilitador de la acreditación e implementación de mecanismos de aseguramiento de la calidad el compromiso de las autoridades. También, se identifica como factor que podría dificultar la institucionalización de dichos mecanismos, la resistencia de los académicos a la evaluación y al cambio.

Lo anterior describe el contexto en el cual las universidades seleccionadas para este estudio han participado en procesos de acreditación institucional, enfrentando el desafío de incorporar mecanismos internos que garanticen la calidad de sus servicios. El análisis de la información recopilada no solo caracteriza con mayor detalle lo que ha ocurrido con la gestión institucional, docencia de pregrado y mecanismos de aseguramiento de la calidad, sino que permite además reconocer cómo distintos tipos de universidades han reaccionado de diferente manera a este mecanismo, adaptándose con mayor o menor éxito a las demandas de los nuevos tiempos. Esta caracterización otorga ciertas luces sobre cuáles elementos -políticas, mecanismos, acciones- incorporados por las instituciones han resultado más comúnmente en transformaciones exitosas, mejorando los resultados de acreditación y, por ende, constatando la presencia de mecanismos eficaces de aseguramiento de la calidad. 


\section{Estrategia metodológica empleada en el estudio}

\section{La estrategia metodológica empleada en el estudio contempla dos etapas:}

\section{Primera etapa}

Se propuso identificar y caracterizar los procesos de consolidación de mecanismos de aseguramiento de calidad en instituciones, procesos incipientes aún no consolidados, y procesos rezagados, dando cuenta del estado actual de cada uno de ellos. Con este propósito se seleccionaron diez universidades, las que se dividieron en tres grupos, considerando la evolución que cada institución ha tenido entre uno y otro proceso de acreditación institucional.

Para dar cuenta del estado de las instituciones en sus procesos de desarrollo del aseguramiento de la calidad, se tuvo en cuenta lo señalado en el artículo 15 de la Ley No 20.219 (que establece un Sistema Nacional de Aseguramiento de la Calidad de la Educación Superior), respecto del objetivo de la acreditación institucional": "evaluar el cumplimiento de su proyecto institucional y verificar la existencia de mecanismos eficaces de autorregulación y de aseguramiento de la calidad al interior de las instituciones de educación superior, y propender al fortalecimiento de su capacidad de autorregulación y al mejoramiento continuo de su calidad" (CNA, 2010b, p.50).

Teniendo en consideración lo señalado en el párrafo anterior, el presente estudio ha considerado que la variación en los años de acreditación institucional otorgados a una universidad entre un proceso y otro, puede ser un buen indicador de mejora de su capacidad de autorregulación, razón por la cual se utilizó este criterio para diferenciar a las instituciones.

3 Todo proceso de acreditación institucional contemplará una autoevaluación interna, una evaluación externa y el pronunciamiento de la Comisión. La acreditación es voluntaria, y en su desarrollo se debe respetar la autonomía de las instituciones; por otra parte, el proceso debe contemplar de forma obligatoria las áreas de gestión institucional y docencia de pregrado. Las demás áreas, de carácter electivo, no serán consideradas en este estudio, por cuanto su foco se encuentra en la gestión institucional. 
De esta manera, primero se seleccionaron las instituciones que, de un proceso de acreditación al siguiente, presentaron mejoras, las que no mejoraron, o incluso retrocedieron en sus resultados en el mismo lapso. La mejora, mantención o retroceso, en este caso, estarán determinados por la variación en los años de acreditación obtenidos por la institución. Luego se realizó una selección de instituciones que presentaron mejoras, diferenciándolas a partir de su situación inicial. Para ello se tomó en consideración la interpretación que la misma CNA elaboró respecto de sus criterios de evaluación, la cual distingue tres tramos en que se pueden ubicar las universidades, según los años de acreditación que obtengan. Esta categorización contempla: $1^{\text {er }}$ Tramo, hasta 3 años; $2^{\circ}$ Tramo, hasta 5 años, y $3^{\text {er }}$ tramo, hasta 7 años (CNA, 2010c).

De esta manera, es posible comprender con mayor claridad que, respecto de las acciones que una institución debe introducir para obtener mejoras en su gestión, el que su punto de partida sea un tramo o el siguiente implicará diferencias sustanciales; es de esperar que, tanto el tipo de mecanismos para incorporar como las dificultades que encuentren en el camino y los elementos que les sean favorables, resulten cualitativamente diferentes. Por ello, el grupo de universidades que presentan mejoras se subdivide entre las que en el segundo proceso de acreditación se mantienen en el primer tramo, y las que pasan al segundo.

Habiendo establecido entonces los criterios utilizados para distinguir las universidades seleccionadas, a continuación se presentan los tres grupos y su composición:

Grupo n. ${ }^{\circ}$ 1: Instituciones con mecanismos de aseguramiento de calidad en desarrollo creciente.

Se consideraron tres instituciones que cumplieran con alguna de las siguientes condiciones: que habiendo participado en un primer proceso de acreditación institucional no hubieran logrado obtener dicha certificación, pero que en un segundo proceso sí se hayan acreditado, por un máximo de 3 años; o que inicialmente hayan acreditado por cierta cantidad de años y que en el segundo proceso 
dicha cantidad haya aumentado, también con un máximo de 3 años. Este grupo es denominado de desarrollo creciente, porque pese a presentar mejoras en todos los casos, se mantiene en el primer tramo de acreditación institucional. Las universidades que integran este grupo se indican en la tabla 3.

Tabla n. $^{\circ}$ 3. Universidades Grupo n. ${ }^{\circ} 1$

\begin{tabular}{|l|c|c|c|c|}
\hline Universidad & $1^{\mathrm{a}}$ acreditación & Año & $2^{\mathrm{a}}$ acreditación & Año \\
\hline Universidad 1 & No & 2005 & Sí $(2$ años $)$ & 2009 \\
\hline Universidad 2 & Sí $(2$ años $)$ & 2007 & Sí $(3$ años $)$ & 2009 \\
\hline Universidad $3^{4}$ & Sí $(2$ años $)$ & 2006 & Sí $(4$ años $)$ & 2008 \\
\hline
\end{tabular}

Grupo n. ${ }^{\circ}$ 2: Instituciones con mecanismos de aseguramiento de calidad consolidados.

Se incluyeron cuatro instituciones que, en un primer proceso de acreditación, se ubicaron en el primer tramo (es decir, que se acreditaran por máximo 3 años), y que en una segunda instancia lograron acreditarse por más años que en la etapa inicial, pasando al segundo tramo definido por la CNA. Estas instituciones se muestran en la tabla 4.

Tabla n. ${ }^{\circ}$ 4. Universidades Grupo n. ${ }^{\circ} 2$

\begin{tabular}{|l|c|c|c|c|}
\hline Universidad & $1^{\text {a }}$ acreditación & Año & $2^{\text {a acreditación }}$ & Año \\
\hline Universidad 4 & Sí (3 años) & 2004 & Sí $(5$ años $)$ & 2007 \\
\hline Universidad 5 & Sí $(2$ años $)$ & 2005 & Sí $(4$ años $)$ & 2007 \\
\hline Universidad 6 & Sí (3 años) & 2005 & Sí (4 años) & 2008 \\
\hline Universidad 7 & Sí (2 años) & 2006 & Sí (4 años $)$ & 2008 \\
\hline
\end{tabular}

Grupo n. ${ }^{\circ}$ 3: Instituciones con mecanismos de aseguramiento de calidad rezagados.

Se seleccionaron 3 instituciones que cumplieran con alguno de los siguientes criterios: que habiendo participado en dos procesos de acreditación, no hayan conseguido la certificación en ninguno de los dos; o que de un proceso a otro hayan disminuido la cantidad de

4 La universidad 3 figura en este grupo y no en el siguiente, porque previo a su acreditación del 2006 participó en otro proceso, el año 2005, en el cual el fallo fue de no acreditación. Sin embargo, por disposiciones legales finalmente adoptadas, no existe un registro público en www.cnachile.cl que refleje esta situación (como un acuerdo de acreditación o de reposición), quedando solo registros en prensa. 
años por los que fueron acreditados. Las universidades en este grupo se resumen en la tabla 5 .

Tabla n. ${ }^{\circ}$ 5. Universidades Grupo n. ${ }^{\circ} 3$

\begin{tabular}{|l|c|c|c|c|}
\hline Universidad & $1^{a}$ acreditación & Año & $2^{\text {a }}$ acreditación & Año \\
\hline Universidad 8 & Sí (4 años) & 2005 & Sí (3 años) & 2009 \\
\hline Universidad 9 & No & 2005 & No & 2007 \\
\hline Universidad 10 & No & 2005 & No & 2007 \\
\hline
\end{tabular}

Cabe señalar que la realidad de una institución que no obtiene la acreditación institucional en dos oportunidades es cualitativamente distinta de la de una universidad que sí se acredita, pero que en una siguiente ocasión obtiene una menor cantidad de años. Sin embargo, en este estudio se decidió considerar a ambos casos en un mismo grupo, ya que ambas situaciones implican la ausencia de mejoras significativas de un proceso a otro y, por ende, operan como contraste respecto de los otros dos grupos; debido a que el objetivo principal en este caso es identificar las prácticas que implican un mejoramiento en la gestión, su ausencia sería el factor común a las universidades del tercer grupo, y que a su vez las diferencia de las demás.

Además de cumplir con el criterio de acreditación, la selección final de universidades considera instituciones de diversa naturaleza, incluyendo públicas, privadas con aporte estatal y privadas autónomas, y de distinta ubicación geográfica. Se tomó la decisión de incluir esta diversidad institucional en la muestra para evitar que las conclusiones que se obtuvieran fueran sesgadas, lo que podría haber ocurrido, por ejemplo, si se hubieran seleccionado únicamente universidades privadas o ubicadas en la región metropolitana.

El criterio basado en los acuerdos de acreditación implica la existencia de un límite temporal en este estudio, debido a la realización de un análisis comparativo entre un primer proceso de acreditación institucional, un segundo proceso y la situación actual. La delimitación temporal comprende los años 2004-2005 -cuando se desarrollaron los primeros procesos- hasta mediados del año 2010, periodo en que se recolectaron los acuerdos de acreditación institucional y comenzó el levantamiento de información. Esto implica que si alguna de estas instituciones concluyó un tercer proceso de acreditación, que hubiera 
sido publicado hacia fines del 2010 o después, dicho acuerdo no ha sido considerado como parte de esta investigación.

El estudio de la realidad de estas diez instituciones se realizó utilizando dos fuentes. La primera, los acuerdos de acreditación institucional publicados en la página web de la Comisión Nacional de Acreditación (www.cnachile.cl), que corresponden a la declaración pública que la Comisión realiza respecto de los resultados de dichos procesos, y que contiene una descripción general de las fortalezas y debilidades de cada universidad respecto de su gestión institucional y su docencia de pregrado, además de las otras áreas que voluntariamente quieran acreditar'; habiendo pasado todas las instituciones seleccionadas por al menos dos procesos de acreditación institucional. Con este fin se analizaron en total 20 acuerdos de acreditación, más algunos acuerdos que daban respuesta a recursos de reposición interpuestos por las universidades.

La otra fuente que se utilizó para recolectar información de estas universidades fue un cuestionario autoaplicado, el que fue difundido y contestado en formato digital. Este estuvo orientado a obtener información respecto de la existencia de políticas, mecanismos y/o procedimientos de aseguramiento de la calidad en las instituciones (años de entrada en vigencia, etc.), así como también sobre las formas en que existen o se expresan dichas políticas, sobre todo con relación a sus mecanismos internos de aseguramiento de la calidad. Este cuestionario consideró como tiempo referencial los últimos 5 años, en los cuales las universidades fueron sometidas a procesos de acreditación institucional.

La aplicación del instrumento fue de carácter intencionado, ya que se realizó en una muestra de autoridades seleccionadas por su vinculación con el tema del aseguramiento interno de la calidad, y por su participación en la toma de decisiones sobre gestión y docencia; rector, aunque en algunos casos se optó por el prorrector, vicerrector

5 En este caso no se consideraron en el análisis las referencias a la investigación, docencia de posgrado, infraestructura y equipamiento u otras áreas no obligatorias de acreditación institucional, centrándose exclusivamente en las áreas obligatorias de gestión y docencia de pregrado. 
académico, encargado de Planificación, encargado de Aseguramiento de Calidad y dos decanos, que dirigen facultades con mayor número de carreras acreditadas.

La tasa de respuesta a esta encuesta fue bastante baja, debido a su extensión y a otros estudios similares que requerían su atención al mismo tiempo, según fue reportado. Sin embargo, se contó con al menos un cuestionario respondido por casi todas las universidades ${ }^{6}$, lo que permitió contrastar la información obtenida desde los acuerdos de acreditación institucional, con lo obtenido a partir de este instrumento. Así, el análisis que se presenta más adelante en este informe corresponde al contraste de ambas fuentes de información, el que permitió generar una imagen global respecto de la situación de cada institución y cada grupo.

Segunda etapa

Tuvo como objetivo identificar buenas prácticas asociadas a la implementación exitosa de mecanismos de aseguramiento de la calidad, al interior de las IES. Para dar cuenta de esto y desarrollar un análisis más profundo y detallado se seleccionaron: Universidad 4, Universidad 6, Universidad 7, Universidad 1.

Las tres primeras universidades pertenecen al grupo denominado instituciones consolidadas, mientras que la última es parte del primer grupo de universidades, en proceso creciente de consolidación. Por ello, se esperaba encontrar en estas instituciones claras evidencias de cambios sustantivos en la gestión, así como transformaciones significativas con relación al aseguramiento de la calidad, las que serían observables durante el periodo que se inicia con el primer proceso de acreditación.

La metodología mediante la cual se desarrolló esta etapa es el estudio de caso. Específicamente, estos fueron desarrollados a partir de entrevistas a informantes calificados de cada institución. El análisis generó una lista de buenas prácticas y su descripción, a partir del cual emanaron las conclusiones del estudio.

6 Solo desde la Universidad 8 no se recibió ninguna respuesta. 
Los informantes calificados de las cuatro universidades seleccionadas en esta etapa son autoridades de cada una de las instituciones vinculadas con el objeto del estudio.

\section{Reflexiones que surgen de la primera fase del estudio}

Teniendo en consideración que esta etapa del estudio constituyó una primera aproximación al tema, los resultados obtenidos permitieron establecer conclusiones que, debido al tamaño de la muestra y al método de investigación, no hacen posible generalizar o realizar afirmaciones taxativas; no obstante, constituyen un buen inicio para poder identificar factores asociados al éxito de la implementación de mecanismos de aseguramiento de la calidad, los cuales son analizados en mayor profundidad en la segunda etapa de este análisis.

En los tres grupos de instituciones se identificó como uno de los cambios más visibles la instalación de unidades técnicas de apoyo a procesos de acreditación. Si bien todas las instituciones las señalan como un cambio relevante asociado a la gestión, parecen existir otros factores que intervendrían en la eficacia de estas unidades. En las instituciones más consolidadas, la implementación de estas oficinas está también asociada a la capacitación de personal técnico de apoyo y a la voluntad política de las autoridades directivas de la universidad, lo que se expresa, entre otras cosas, en la asignación de un presupuesto específico para desarrollar estas actividades.

Al parecer, otro de los factores determinantes en la acreditación es la operacionalización de la misión en metas y objetivos verificables. Los dos grupos menos consolidados de instituciones dan cuenta de que han realizado ajustes y/o cambios en la forma de plantearse sus misiones institucionales, sin embargo, el estado de operacionalización de las mismas parece ser bastante heterogéneo, lo que se podría relacionar con el resultado de las acreditaciones obtenidas. Se puede inferir que en la medida que las instituciones pasan de una misión declarativa y/o genérica, a una en la cual se establecen claramente prioridades y mecanismos de verificación, se generaría una mayor sintonía entre políticas y mecanismos internos y resultados obtenidos. 
Asociado con lo anterior, la planificación institucional y su vinculación con los procesos de acreditación parece ser otro de los factores que reflejan mayor afianzamiento en los procesos de gestión. El grupo de instituciones más consolidadas señala que, si bien los mecanismos de planificación estratégica existían previamente a la acreditación, esta posibilitó perfeccionarlos, al tiempo que también se comenzaron a obtener resultados coherentes con metas y objetivos definidos. Por su parte, las instituciones no consolidadas reconocen que sus mecanismos de planificación aun son deficitarios y que no existen metas y/o indicadores que den cuenta del avance de la misión.

Respecto de la estructura organizacional, los dos grupos de instituciones menos consolidadas dan cuenta de ajustes y cambios en su estructura, señalando que se incorporaron nuevas instancias y se readecuaron otras. Según la opinión de las instituciones, estos cambios están relacionados con una mejor distribución de tareas y coordinación interna. Para el grupo de las instituciones consolidadas no se reportan grandes ajustes en la estructura, sino más bien los cambios se relacionan con la gestión interna, pasando de una centralizada a una donde existen espacios de mayor participación y desconcentración en los procesos de toma de decisiones estratégicas, además de cambios en la gestión financiera y asignación de recursos. Se puede decir que los procesos de gestión en este tipo de instituciones tienden a profesionalizarse y alinearse con los objetivos estratégicos definidos por las mismas.

La participación parece también tener un rol determinante en la eficacia de los mecanismos de aseguramiento de la calidad; todas las instituciones señalan que existe un mayor grado de involucramiento de los actores internos (estudiantes y profesores), la diferencia parece estar en el grado en que estos actores participan en la gestión cotidiana de la institución. En este sentido, mientras en el grupo de instituciones consolidadas se establecen mecanismos formales de participación, y se incorporan las opiniones de los actores internos y externos en los procesos de toma de decisiones; en el grupo de las menos consolidadas, la participación se limita a la recolección de opinión de los académicos, no existiendo ningún mecanismo para actores externos como egresados y empleadores. 
Respecto de la gestión de la docencia, los resultados parecen ser menos evidentes o tener un menor impacto. No obstante, existen aspectos identificados por todas las instituciones que podrían dar cuenta de factores asociados al éxito vinculados con los procesos de acreditación:

El primero está relacionado con la existencia de un modelo educativo y/o curricular implementado institucionalmente. Si bien este no es un criterio explícito de la CNA, los acuerdos revelan que es un factor analizado a la hora de asignar los años de acreditación.

Las instituciones más consolidadas declaran tener modelos educativos que se aplican sistemáticamente en todos los niveles institucionales, unido a la incorporación de mecanismos de aseguramiento de la calidad para sus programas, un mayor control sobre las políticas de docencia y cambios curriculares. En el grupo de instituciones intermedias, si bien se declara la existencia de modelos, estos no se aplican sistemáticamente; y por último, en el grupo de instituciones no consolidadas no existe un modelo único ni políticas explícitas.

Otro punto significativo, y que solo fue mencionado por las instituciones consolidadas, tiene relación con el financiamiento asignado a la docencia. En este grupo de instituciones se señala que hay un incremento de recursos, el que podría estar vinculado con mejores logros en la acreditación y una docencia más consolidada o con mejor resultado.

Es importante destacar que los aspectos reportados por las instituciones, así como las dimensiones identificadas en los acuerdos de acreditación, son bastante coherentes con los resultados internacionales sobre evaluación de impacto. Por otro lado, si bien los resultados obtenidos en esta primera parte del estudio no son definitorios, permiten identificar una serie de factores y/o indicadores vinculados con el éxito en los procesos de acreditación institucional. Estos elementos resultan de gran utilidad para el desarrollo de la segunda parte de esta investigación, ya que facilitan la elaboración de pautas de entrevista adecuadas, que profundicen en los aspectos necesarios para la identificación de buenas prácticas en la gestión institucional. 
- Buenas prácticas en la gestión universitaria

La segunda fase de la investigación consistió en el desarrollo de 16 entrevistas a informantes calificados de las cuatro universidades seleccionadas. Como se señaló previamente, estas instituciones fueron seleccionadas de la muestra inicial, en función de sus avances en la gestión y el aseguramiento de la calidad. Los entrevistados fueron vicerrectores u otros directivos, vinculados con las áreas de interés del estudio. Esta fase estuvo destinada a identificar buenas prácticas en diversas áreas de la gestión institucional, focalizándose las que se señalan a continuación:

- Buenas prácticas asociadas a la misión institucional

Una de las primeras prácticas que se incorporan en las instituciones es el ajuste de sus misiones y objetivos institucionales. Las autoridades reportan que en una primera instancia, previa a la incorporación de mecanismos de aseguramiento de la calidad y a la acreditación, las misiones institucionales eran declarativas. Posteriormente a la incorporación de mecanismos de aseguramiento de la calidad, las instituciones habrían revisado el planteamiento de sus visiones y misiones, traduciéndolas en metas y objetivos concretos y susceptibles de medición.

En la mayoría de los casos estudiados, esto permitió replantearse el quehacer institucional, trayendo como consecuencia otros cambios que repercutieron directamente en la gestión.

Es así como muchos de los cambios y ajustes posteriores realizados se vinculan con la existencia de una misión y visión más funcionales, que orientan y permiten tener un contexto para las actividades que la universidad pretende lograr.

- Buenas prácticas en la estructura de las organizaciones

En todas las instituciones analizadas se reporta como práctica que ha favorecido la gestión, la creación y/o ajuste de organismos en las estructuras de gobierno. 
Los cambios que se mencionan son, por ejemplo, la creación de unidades encargadas de realizar los procesos de análisis institucional, planificación estratégica y aseguramiento de la calidad. Con relación a esto último, es notoria la mayor visibilidad y funcionalidad de ciertas actividades institucionales definidas como prioritarias, como la revisión de los propósitos y la misión institucional.

El rol de estas oficinas es, fundamentalmente, asesorar a las instancias directivas en los procesos de toma de decisiones. Además, con ellas se incorporan unidades internas encargadas del control de la gestión, el seguimiento de planes estratégicos y de acciones de mejoramiento y evaluación basada en indicadores (deserción, retención, etc.).

Respecto de las características internas de estas unidades, en su mayoría se encuentran en las vicerrectorías, o bien -según la función específica que corresponda y el estilo de gestión de la universidaden la rectoría, como órgano asesor del rector, están conformadas por equipos multidisciplinarios que han sido capacitados en las funciones específicas que desarrollan.

Lo anterior permite inferir que son unidades estratégicas que colaboran con la gestión de la institución; cuentan con el personal técnico necesario para desarrollar su función y suficiente apoyo y autonomía para llevar a cabo los procesos de evaluación y seguimiento de las metas y políticas institucionales. Cabe destacar que la función que desarrollan estas unidades deja de ser meramente técnica con respecto de la conducción y desarrollo de procesos de acreditación. Si bien esta función sigue existiendo, se encuentra ubicada en otro nivel (Vicerrectoría Académica) o bien forma parte de la dirección, siendo una de sus funciones, pero no la principal.

\section{- Buenas prácticas relacionadas con la descentralización de la gestión}

En esta dimensión, los cambios observados se vinculan con dos aspectos principales. El primero -tratado con mayor profundidad en el punto anterior-, relacionado con la práctica de adecuar la estructura organizacional, reflejado en la creación de unidades de apoyo técnico que soportan el trabajo de la gestión en las direcciones 
superiores. Esto permite, por una parte, descentralizar los procesos de toma de decisiones, y por otra, profesionalizar los procesos de gestión institucional.

Por otro lado, la visibilidad que han alcanzado los procesos y mecanismos de aseguramiento de la calidad al interior de las universidades, revela la incorporación de prácticas que han generado cambios en las culturas internas de dichas instituciones. En este sentido, los entrevistados reportan una mayor participación y compromiso de los actores, tanto internos como externos, vinculados con los procesos de formación.

Este cambio cultural en la gestión permite una mayor apertura a los procesos de evaluación, donde la opinión de los actores internos (estudiantes y profesores), así como, en menor medida, de los externos (empleadores y egresados), juega un rol fundamental en los procesos de toma de decisiones institucionales. Se observa también una promoción interna a la participación en procesos de autoevaluación, de planes de desarrollo institucional, entre otros. Este mayor grado de participación se expresa en un mejor entendimiento de metas y objetivos institucionales y más colaboración para su cumplimiento.

\section{- Buenas prácticas sobre la formalización de políticas y} mecanismos institucionales

Otra práctica que se incorpora es la generación de un mayor grado de ordenamiento interno, traducido en mayor claridad en la especificación de funciones y roles, redefinición de las dinámicas internas de procesos de toma de decisiones y mayor profesionalización de las funciones de gestión y análisis institucional.

Luego de experimentar procesos de acreditación, las instituciones tienden a alinear la definición de su misión institucional, y las acciones y mecanismos internos para su cumplimiento. En la mayoría de los casos se evidencia la existencia de mecanismos que regulan determinadas funciones institucionales, pero que no están formalizados para asegurar su aplicación eficaz en todos los niveles institucionales. Uno de los primeros cambios evidenciados es una 
mayor formalización de las prácticas internas y la creación de una reglamentación que regule su aplicación.

Asimismo, se observa una revisión de la adecuación de las políticas internas y su relación con el proyecto de desarrollo institucional, generando cambios internos que permiten tener una mayor coherencia y alineación entre políticas, mecanismos y acciones institucionales. Esta formalización facilitó un mayor grado de cumplimiento de los propósitos institucionales.

- Buenas prácticas asociadas a la función de planificación institucional y gestión financiera

La disponibilidad de recursos y su adecuada administración es una de las preocupaciones centrales en todas las IES. La aplicación de mecanismos de aseguramiento de la calidad en el ordenamiento institucional y de funciones de gestión de las universidades generó una mejora en general. En lo administrativo y financiero, promovió como práctica el desarrollo de procesos más eficientes y eficaces, y contribuyó a que las decisiones financieras se adoptaran sobre la base de resultados de evaluación, sistemáticos y validados, obtenidos mediante la evaluación externa.

Existe una mayor correlación entre los procesos de asignación de recursos y las prioridades establecidas en la misión y propósitos institucionales. Esto se relaciona con una redefinición de las funciones universitarias, específicamente en lo que se refiere a la valorización de las características de la docencia y sus resultados, y a la institucionalización de los procesos de evaluación pedagógica, generando más inversión en esta área.

\section{- Buenas prácticas en la gestión de la información}

A partir de la aplicación de mecanismos de aseguramiento de la calidad se introdujo como práctica la generación y sistematización de información o datos institucionales sobre las distintas funciones universitarias.

La instalación de dichos mecanismos obligó a la generación y ordenamiento de información válida y confiable al interior de las 
instituciones. Si bien esta sistematización y ordenamiento interno surgió como respuesta a las demandas externas provenientes de los procesos de acreditación, a medida que estos se fueron consolidando, la información institucional tomó un rol principal en la gestión interna, constituyéndose en un pilar fundamental.

El mejoramiento en los procesos de gestión de la información fue acompañado por la incorporación de herramientas tecnológicas ad hoc a las necesidades de las instituciones; es así como muchas de ellas desarrollan sus propias plataformas computacionales para sistematizar los datos. Otras adquirieron programas específicos para el apoyo de los procesos de control y gestión interna, y capacitaron a las distintas unidades para utilizar estos sistemas; en algunos casos adaptaron lo existente con el objetivo de hacerlos más eficientes y adecuados a las demandas internas.

También, las instituciones tendieron a centralizar los sistemas de información y a integrarlos en una plataforma única, que les permitiese gestionar la información de los distintos niveles. Así, se incorporaron a la gestión institucional los resultados individuales de cada una de las unidades académicas, tanto en lo que se refiere a los aspectos financieros de las mismas como en los aspectos académicos y administrativos.

Esta incorporación de herramientas tecnológicas generó un cambio en la cultura institucional, pasando de procesos de toma de decisiones intuitivos y segregados entre una unidad y otra, a procesos basados en evidencias.

\section{- Buenas prácticas en la gestión académica institucional}

Respecto de los procesos académicos, en las instituciones analizadas la aplicación de mecanismos de aseguramiento de la calidad tiene un acento especial en las formas en que se organiza la función docente. Por consiguiente, es posible observar que parte de las prácticas incorporadas se relacionan con la forma que desarrollan sus procesos de diseño y ajuste curricular. 
El primer aspecto que se observa es la institucionalización de los procesos de revisión y ajuste de la estructura curricular, en la adecuación de perfiles de egreso y en mecanismos para verificar su cumplimiento. Asimismo, se observan cambios en los mecanismos para evaluar y mejorar la disponibilidad e idoneidad de recursos humanos, infraestructura y recursos de aprendizaje de manera acorde a las necesidades de los programas.

Algunos factores interesantes en que se reflejan estos cambios se relatan a continuación:

- Revisión de la oferta académica (programas y sedes) y ajuste a criterios de evaluación. Existe mayor preocupación por la homologación de la oferta y pertinencia de la misma.

- Mejora de la dotación docente: redefinición del perfil académico en función de los propósitos de cada programa.

- Fortalecimiento de los servicios de apoyo a la enseñanza.

- Instauración de modelo educativo institucional, cambios curriculares y alineamiento con las políticas institucionales.

- Acreditación de carreras, decisión derivada de la conciencia que los procesos de acreditación institucional y de carreras son complementarios.

\section{Reflexiones y recomendaciones}

La investigación hasta aquí presentada permitió alcanzar diversos objetivos, según fueron planteados desde un comienzo. A partir del análisis de los acuerdos de acreditación institucional, sumado a la información obtenida mediante encuestas, se caracterizaron los procesos de cambio al interior de las universidades que se generan a partir de la acreditación institucional, identificando además los ámbitos de la gestión en los que ocurren las principales transformaciones. Además, desde la profundización en algunos casos considerados como ejemplares, fue posible identificar una serie de buenas prácticas o acciones en las cuales se sustentaron los avances institucionales, con el objetivo de difundir esta información, potencialmente útil, entre los actores del medio universitario y del sistema de educación superior chileno en general. 
Aparte de identificar los ámbitos específicos en que se producen cambios, y el sentido en que ellos ocurren, la investigación realizada permitió identificar la existencia de elementos comunes a las universidades que se encuentran en un mismo estadio, y diferentes entre instituciones con desarrollo incipiente y consolidado. Este hecho pareciera indicar la existencia de una senda evolutiva en los procesos de acreditación institucional, es decir, un recorrido por el cual todas estas instituciones parecen transitar, aunque a distintos ritmos.

A continuación se detallan los elementos que componen este recorrido.

El primer aspecto fundamental en la evolución de los mecanismos de aseguramiento de la calidad es la revisión de la misión y la incorporación de esta en los procesos de planeación interna. Si bien este aspecto pudiese parecer básico, en su debido momento las instituciones analizadas debieron realizar un proceso de análisis profundo respecto de las implicancias, significado y alcance de la misión; por ello, esta revisión es considerada como una fase fundamental en la incorporación de mecanismos de aseguramiento de la calidad en las universidades.

En la mayoría de los casos, las misiones institucionales corresponden a los propósitos declarados de lo que pretenden lograr. No obstante, se observa que el proceso de resignificación y análisis de la misión estuvo acompañado de la identificación de ejes o propósitos estratégicos, con lo que se reconocieron metas u objetivos concretos y medibles. En este sentido, las instituciones operacionalizaron sus misiones con el objetivo (explícito y/o implícito) de usarlas en la práctica como instrumentos de la gestión. Además, en la actualidad, las universidades que presentan un mayor nivel de consolidación respecto de su gestión interna de la calidad, cuentan con mecanismos formales y sistemáticos para su evaluación.

Aunque la relevancia que adquiere el ajuste de la misión se debe principalmente al modelo de acreditación utilizado, en el que es un eje central, las acciones que posteriormente realizan las instituciones parecen estar relacionadas con los modelos de gestión de la calidad interna en cada una. 
Otra característica que se identifica al respecto es que en los primeros procesos de reacreditación la revisión de la misión habría sido efectuada principalmente en función de los criterios de evaluación externa, y en el contexto de dicho proceso. Posteriormente a esa primera acreditación, donde el resultado pudo ser positivo o no, la revisión estuvo más bien centrada en el proyecto de desarrollo institucional de la universidad. Así, es posible observar que las universidades deciden potenciar ciertas funciones, dándoles mayor importancia dentro de las actividades que se realizan; o bien, incorporar algunas que, si bien estaban declaradas, no tenían un desarrollo significativo, especialmente la investigación, el posgrado y la vinculación con el medio.

Particularmente en las instituciones que tienen más de una sede, surgen una serie de acciones tendientes a regular la oferta académica de las mismas. Además, se definen prioridades de crecimiento, y se establecen protocolos claros para su implementación y desarrollo. Todos estos procesos se encuentran acompañados de cambios en la estructura de gestión, sobre todo en lo directivo.

Otro aspecto que se identifica claramente es que, luego de la revisión de la misión, las instituciones entran en un proceso de formalización de su normativa y reglamentos internos, y se crean nuevas normas y procedimientos más acordes a las actividades que realizan. Este punto también se observa como una evolución entre un proceso de acreditación y otro: en una primera fase, ocurre una formalización de políticas y mecanismos institucionales, traducida en ajustes en los reglamentos internos, y en la creación de nueva normativa. Luego, en una segunda etapa, las instituciones se concentran en su cumplimiento y focalización, e introducen mecanismos internos de control y seguimiento. Por último, en una tercera etapa, las instituciones que presentan una mayor consolidación de sus sistemas de gestión de la calidad evolucionan desde la incorporación de mecanismos de control a la de componentes de administración y de gestión más participativos, en los cuales los procesos de toma de decisiones involucran a la comunidad académica. 
Una de las figuras que emerge con más fuerza como cambio o acción que favorece la gestión interna de la calidad es la creación de oficinas de análisis institucional. Aun cuando todas las instituciones analizadas cuentan con unidades que cumplen esta función, al parecer su alcance y las funciones que desarrollan parecen no ser iguales.

El primer aspecto para analizar es la posición que estas oficinas ocupan en la estructura organizacional. Las universidades que tienen una mayor consolidación en la aplicación de mecanismos de aseguramiento de la calidad, tienden a ubicar estas unidades a nivel directivo. Son unidades que desarrollan la función de análisis interno, y en las que los procesos de acreditación son una de sus actividades. Los profesionales que las integran conforman un equipo técnico, especializado en procesos de control de gestión, planificación y acreditación. Además, disponen de mecanismos de seguimiento y gestión de observaciones resultantes de los procesos de acreditación institucional y de carreras.

Por otro lado, las instituciones que recientemente han incorporado mecanismos de aseguramiento de calidad cuentan con unidades con un perfil más orientado a gestionar los procesos de acreditación; son unidades de apoyo, en la mayoría de los casos dependientes de alguna de las vicerrectorías. Por otra parte, en estas instituciones la función de planeamiento institucional está ubicada en otra instancia de la estructura. La unidad de análisis está conformada por un equipo reducido (no más de tres personas) y su rol principal es acompañar a las unidades académicas en los procesos de autoevaluación.

Por último, se observan también diferencias importantes en la manera en que las instituciones gestionan sus indicadores internos de calidad. En las universidades que se encuentran en una etapa inicial en este aspecto, las principales acciones están destinadas a desarrollar sistemas integrados de información, que les permita tomar decisiones basadas en evidencias. No se utilizan indicadores referenciales, sino más bien se sistematizan los resultados obtenidos en las distintas áreas, se mide la deserción, retención, etc. En las 
instituciones más consolidadas se observa una mayor gestión de indicadores, traducida en acciones concretas, por ejemplo para mejorar la deserción, retención, entre otros; además de la medición de la efectividad de estas acciones.

De esta manera, es posible establecer el recorrido que realizarían las universidades estudiadas, en la medida en que participan de sucesivos procesos de acreditación institucional. Primero, las instituciones evalúan y corrigen la definición de su misión, y la orientan en función de metas y objetivos, e incorporan maneras de medir su logro. Luego, las universidades se abocan a afinar su normativa, sus reglamentos y procedimientos asociados, como formalización de sus políticas y mecanismos, particularmente en lo que respecta a la gestión de la calidad. Culmina este proceso, la etapa de consolidación de un organismo orientado a mantener y desarrollar el análisis institucional, función relacionada directamente con el aseguramiento interno de la calidad.

Sobre este proceso es posible agregar dos elementos de gran relevancia. Este recorrido estaría directamente relacionado con el grado de éxito en la acreditación institucional, habría una asociación entre la cantidad de años otorgados en un proceso particular, y la etapa de evolución en la que se encuentra la universidad. La sucesión se desarrolla en la medida en que las instituciones van incorporando mecanismos de aseguramiento de la calidad, la senda parte desde la acreditación como proceso externo, cuando la institución reacciona al mismo, hasta que estos mecanismos han sido incorporados internamente, y el aseguramiento de la calidad ha llegado a ser preocupación y práctica propia de la universidad. Esto indicaría que para las universidades que buscan mejorar sus resultados de acreditación institucional, una estrategia adecuada sería incorporar el aseguramiento de la calidad no solo en sus actividades, sino también en metas, objetivos y en su cultura. En la medida en que la búsqueda de la calidad sea un valor asumido como propio por la comunidad, y llevado a todos los ámbitos de la gestión universitaria, esto se verá reflejado en sus procedimientos y prácticas, y por ende en sus resultados de acreditación. 
Para concluir, cabe señalar que este estudio que da cuenta de los objetivos formulados en sus inicios, contribuye a la comprensión del sistema universitario actual y permite identificar ciertos elementos que orienten futuras investigaciones. No obstante, este esfuerzo no pretende ni logra agotar el tema de la gestión institucional y los mecanismos de aseguramiento de la calidad, sino más bien abre nuevas interrogantes al respecto.

Algunas temáticas que quedan pendientes para futuros estudios son, entre otras, la incidencia y posibles diferencias de distintos actores de la comunidad universitaria -académicos, estudiantes, administrativos, directivos- en la incorporación de estos mecanismos; de qué manera la decisión de acreditar es asumida por los distintos componentes de la estructura, y las dificultades que la institución encuentra para su difusión y compromiso real; o la variación en los resultados de la gestión institucional -como también del proceso formativo- medida en indicadores, que permitan cuantificar los efectos; la gestión de institutos profesionales y centros de formación técnica a partir de la incorporación de estos procesos. Con relación a otros aspectos más allá de la gestión, resultaría de gran relevancia estudiar la incidencia que los mecanismos de aseguramiento de la calidad tienen en la docencia universitaria. Es de esperar que estas interrogantes, así como otras que puedan surgir, sean abordadas en futuras investigaciones, con el fin de mejorar el conocimiento disponible respecto del sistema de educación superior chileno.

\section{Referencias bibliográficas}

Brennan, J., y Shah, T. (2000). Quality assessment and institutional change: Experiences from 14 countries. Higher Education, vol.40.

CINDA (2010). Proyecto Alfa. Obtenida de Aseguramiento de la Calidad: Políticas Públicas y Gestión Universitaria: http://www.cinda.cl/ proyecto_alfa/index.htm

CNA (2010a). Comisión Nacional de Acreditación. Obtenido de http://www. cnachile.cl

CNA (2010b). Memoria Institucional CNA-Chile 2007-2010. Santiago: CNA. 
CNA (2010c). Operacionalización criterios de evaluación en procesos de acreditación. Documento aprobado para publicación en sesión del 11 de agosto de 2010. Obtenida de http://www.cnachile.cl/wp-content/ uploads/2010/10/Op-Criterios-Acreditaci\%C3\%B3n.pdf

Frederiks, M. M., Westerheijden, D. F., y Weusthof, P. J. (1994). Effects of Quality Assessment in Dutch Higher Education. European Journal of Education 29.

Harvey, L. (2006). Impact of quality assurance: overview of a discussion between representatives of external quality assurance agencies. Quality in Higher Education, vol 13, n. ${ }^{\circ} 3$.

Harvey, L., y Newton, J. (2004). Transforming quality evaluation: moving on. Dynamics and effects of quality assurance in higher education - various perspectives of quality and performance at various levels (pp. 1-25). Douro: Fundação das Universidades Portuguesas.

IPSOS (2010). Estudio exploratorio sobre efectos de la acreditación institucional en la calidad de la educación superior en Chile. Santiago: Estudio elaborado para CNA.

Lemaitre, M. J. (2005). Aseguramiento de la calidad en Chile: impacto y proyecciones. Serie Seminarios Internacionales - Consejo Superior de Educación, pp. 55-69.

Lester, S. (2005). Assessing the self-managing learner: A contradiction in terms? En L. Cunningham, D. O'Reilly, y S. Lester, Developing the capable practitioner (pp. 99-108). Kogan Page.

Stensaker, B. (2008). Outcomes of quality assurance: A discussion of knowledge, methodology and validity. Quality in Higher Education, $\operatorname{vol} 14,{ }^{\circ}{ }^{\circ} 1$. 